\title{
ZONIFICACIÓN GEOMORFOLÓGICA PARA LA EVALUACIÓN DE LA SUSCEPTIBILIDAD A LOS DESLIZAMIENTOS EN LA CUENCA DEL RÍO VIEJO, PURISCAL, COSTA RICA
}

\author{
GEOMORPHOLOGICAL ZONATION FOR THE ASSESSMENT OF LANDSLIDE \\ SUSCEPTIBILITY IN THE VIEJO RIVER BASIN, PURISCAL, COSTA RICA
}

\author{
Ángela M. Suárez ${ }^{1 *}$, Giovanni Peraldo², Elena Badilla² \& Luis G. Obando² \\ ${ }^{1}$ Posgrado Centroamericano en Geología, Apdo. 214-2060, Universidad de \\ Costa Rica, San José \\ ${ }^{2}$ Escuela Centroamericana de Geología, Apdo. 214-2060, Universidad de \\ Costa Rica, San José \\ *Autora para contacto: marcela.suarez@gmail.com
}

(Recibido: 22/05/09; aceptado: 12/12/09)

\begin{abstract}
A landslide inventory and a detailed geomorphological analysis were done, focusing on the identification of mass movement related morphologies, and with the purpose of making a preliminary landslide susceptibility analysis of the Viejo river basin, Puriscal. From a geomorphological point of view, denudational units are predominant. They include regions severely affected by mass movements and represent $92,3 \%$ of the basin area. Geomorphology proved to be an important conditioning factor for landslide susceptibility and a valuable instrument for the interpretation of historical dynamics of the geoforms and past landslide events, with the aim of validating the variables and methodologies of susceptibility zonation to be considered.

Keywords: inventory, zonation, susceptibility, landslide, drainage, geomorphology, Viejo river, Puriscal.

RESUMEN: Se realizó un inventario de deslizamientos y un análisis geomorfológico detallado, con énfasis en la identificación de las morfologías relacionadas con los movimientos en masa, con el fin de hacer un análisis preliminar de la susceptibilidad a deslizamientos para la cuenca del río Viejo, Puriscal. En la geomorfología de la cuenca, sobresalen unidades de origen denudacional que abarcan el 92,3\% del área de la cuenca y entre las que se encuentran áreas severamente afectadas por movimientos en masa. La geomorfología del área demuestra la importancia de su inclusión como factor condicionante en el estudio de la susceptibilidad, y como instrumento para interpretar la dinámica histórica de las formas en el área y los eventos de deslizamiento que se han presentado, con el fin de validar las variables y metodologías de zonificación de susceptibilidad a tener en cuenta.

Palabras clave: Inventario, zonificación, susceptibilidad, deslizamientos, drenaje, geomorfología, río Viejo, Puriscal.
\end{abstract}




\section{INTRODUCCIÓN}

La identificación y zonificación de las amenazas forman parte de las primeras y más importantes labores en el contexto de prevención y mitigación en la gestión del riesgo, y aunque actualmente hay diversas técnicas propuestas para ello, la tarea más difícil está en seleccionar la técnica que permita obtener los resultados más precisos, teniendo en cuenta la información del lugar, la escala de trabajo, la extensión del área de estudio y los recursos disponibles

Para el caso de los deslizamientos, en los últimos años se han propuesto varios métodos y técnicas para analizar los factores que han favorecido o pueden llegar a favorecer su ocurrencia, y para producir mapas de amenaza, que muestran la probabilidad espacial y temporal de ocurrencia, ó por lo menos, mapas de susceptibilidad, que definen únicamente la probabilidad espacial de ocurrencia.

El inventario de deslizamientos es un instrumento clave para el entendimiento de la amenaza; y aunque el mapa que lo representa es considerado por sí mismo como mapa de susceptibilidad, realizado mediante el método directo, éste también es considerado un instrumento clave para aplicar métodos estadísticos y para calibrar los resultados en la zonificación de la susceptibilidad.

El mapa de inventario se basa en la interpretación de fotografías aéreas, trabajo de campo y/o una base de datos sobre la ocurrencia de deslizamientos históricos en el área. El producto final muestra la distribución espacial de los movimientos en masa, representados ya sea a escala o como puntos (Van Westen \& Soeters, 2000).

Por otro lado, la elaboración de un mapa de inventario debe ir de la mano de una caracterización geomorfológica, ya que los factores morfológicos como la geometría de las laderas (grado de pendiente, altura, longitud, forma, aspecto, etc.) y las características de las cuencas (orden, magnitud, gradiente, etc.), reflejan la evolución geomorfológica pasada y presente de una región, y proporcionan información fundamental sobre los procesos de deslizamiento (Carrara et al., 1995).

En Costa Rica, los deslizamientos al igual que las inundaciones, son los sucesos que más afectan a la población debido a su recurrencia e impacto local. En la actualidad aún hay lugares que no cuentan con su respectiva zonificación de amenazas, y en la mayoría de los casos, sólo se han realizado estudios a una escala muy pequeña. Un ejemplo de esto es el Valle Central, que actualmente cuenta con una macrozonificación de la amenaza por deslizamientos, elaborada por Mora et al. (1992) (publicada a escala 1: 286000 ), que aunque constituye una muy buena aproximación del grado de amenazas de deslizamiento, no muestra resultados al detalle que muchas veces se requiere para la toma de decisiones a nivel local.

Asimismo, en Costa Rica al igual que en otros países, los mapas de inventario de deslizamiento son escasos debido a la insuficiencia de recursos para investigación; lo cual limita el conocimiento de los factores y mecanismos que causan los deslizamientos.

El propósito del presente estudio fue realizar un inventario de deslizamientos, y un análisis geomorfológico detallado, con énfasis en la identificación de las morfologías relacionadas con los movimientos en masa, con el fin de hacer un análisis preliminar de la susceptibilidad a deslizamientos, para la cuenca del río Viejo, Puriscal.

Dicho análisis se llevó a cabo a partir de observaciones de campo y de la interpretación de las fotografías aéreas a color del proyecto Terra 1998, escala 1:40 000, y de fotografías aéreas digitales infrarrojas del proyecto CARTA 2005, cuya resolución espacial es de aproximadamente 5-6 m.

Al estudiar la amenaza por deslizamientos en Santiago de Puriscal y alrededores, Peraldo (1996) recomendó hacer estudios más detallados en algunos sectores específicos. Uno de estos sectores corresponde al área abarcada por la cuenca del río Viejo. Es por esta recomendación, y por los procesos de deslizamiento que se han presentado en la cuenca en los últimos años, que fue seleccionada el área de estudio.

Los resultados de este estudio se presentan en dos mapas: El primero corresponde con el mapa de inventario de deslizamientos, cuyo objetivo principal, además de mostrar la distribución de todos los deslizamientos que han dejado morfologías típicas en el área, es ser una herramienta para futuros mapas de densidad ó mapas 
de susceptibilidad realizados mediante métodos estadísticos. El segundo corresponde al mapa geomorfológico, en el que se presenta una visión detallada de la geomorfología del área de estudio, y las principales unidades geomorfológicas.

\section{EL ÁREA DE ESTUDIO}

La cuenca del río Viejo pertenece, desde el punto de vista administrativo, al cantón de Puriscal y, en pequeña proporción, al cantón de Mora (Fig. 1), y abarca un área de $25,2 \mathrm{~km}^{2}$. El río Viejo es uno de los afluentes del río Tabarcia, el cual a su vez irriga la cuenca del río Pirrís, ubicada en el Pacífico Central de Costa Rica.

Puriscal es uno de los cantones más afectados por procesos de remoción en masa en el país. Su ciudad principal, denominada Santiago (Fig. 1), se encuentra ubicada sobre un deslizamiento cuya actividad se conoce desde 1918 (Mora, 1991).

Es importante resaltar que dentro de la cuenca existen poblaciones como San Rafael, Guayabo, Floralia, y algunos barrios de la ciudad

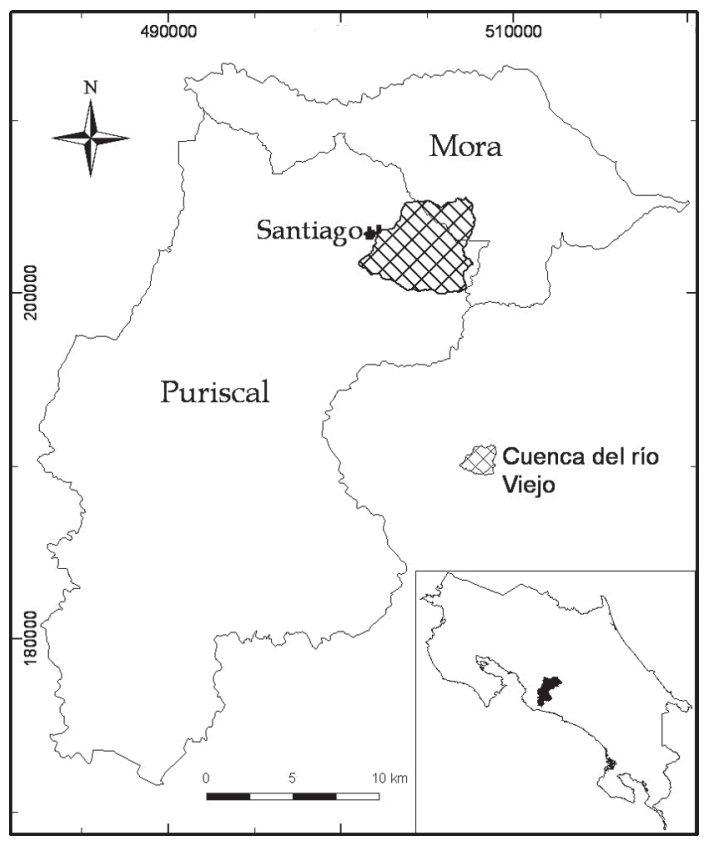

Fig. 1: Ubicación de la cuenca del río Viejo, en un contexto nacional y cantonal. de Santiago; que podrían ser afectados por procesos de remoción en masa (Fig. 2). La población aproximada de esta cuenca es de 10200 habitantes (INEC, 2000).

Desde el punto de vista geológico, la cuenca del río Viejo se encuentra dominada, en un $85 \%$ de su área, por rocas de la Formación Grifo Alto definida por Denyer \& Arias (1991), la cual se compone por rocas volcánicas andesíticas y piroclastos que sobreyacen los depósitos sedimentarios y volcánicos miocénicos del Valle Central. La Formación La Cruz, del Mioceno Superior terminal y está constituida por un conjunto de lavas, predominantemente basálticas, y tobas, que sobreyacen concordantemente la secuencia sedimentaria miocénica según (Denyer \& Arias, 1991). Cubre aproximadamente el 14\% del área de la cuenca. El 1\% restante del área de la cuenca, corresponde con los Depósitos Parálicos de la Formación La Cruz (Denyer \& Arias, 1991), compuestos por paquetes de conglomerados de matriz arenosa y areniscas vulcanoclásticas pardas, con intercalaciones de tobas y tobitas rojas y moradas y estratos esporádicos de lutita carbonosa.

\section{INVENTARIO DE DESLIZAMIENTOS}

De acuerdo con Malamud et al. (2004), los inventarios de deslizamiento generalmente se derivan de la identificación y geo-referenciación de: (1) deslizamientos asociados con un evento disparador específico, y (2) deslizamientos históricos, los cuales son la suma de uno o varios deslizamientos activos o recientemente activos a lo largo del tiempo en una región.

Para este estudio se hizo un inventario de deslizamientos históricos, a partir de entrevistas informales a habitantes de la cuenca, de visitas de campo y de la interpretación de las fotografías aéreas que cubren el área. El trabajo consistió en representar la distribución de los depósitos de deslizamiento y señalar las coronas que eran identificables, de acuerdo con la escala de las fotos. El mapa generado se muestra en la figura 3.

Según Malamud et al. (2004), muchos factores afectan la fiabilidad, la suficiencia y la resolución de un mapa inventario, entre los que pueden 


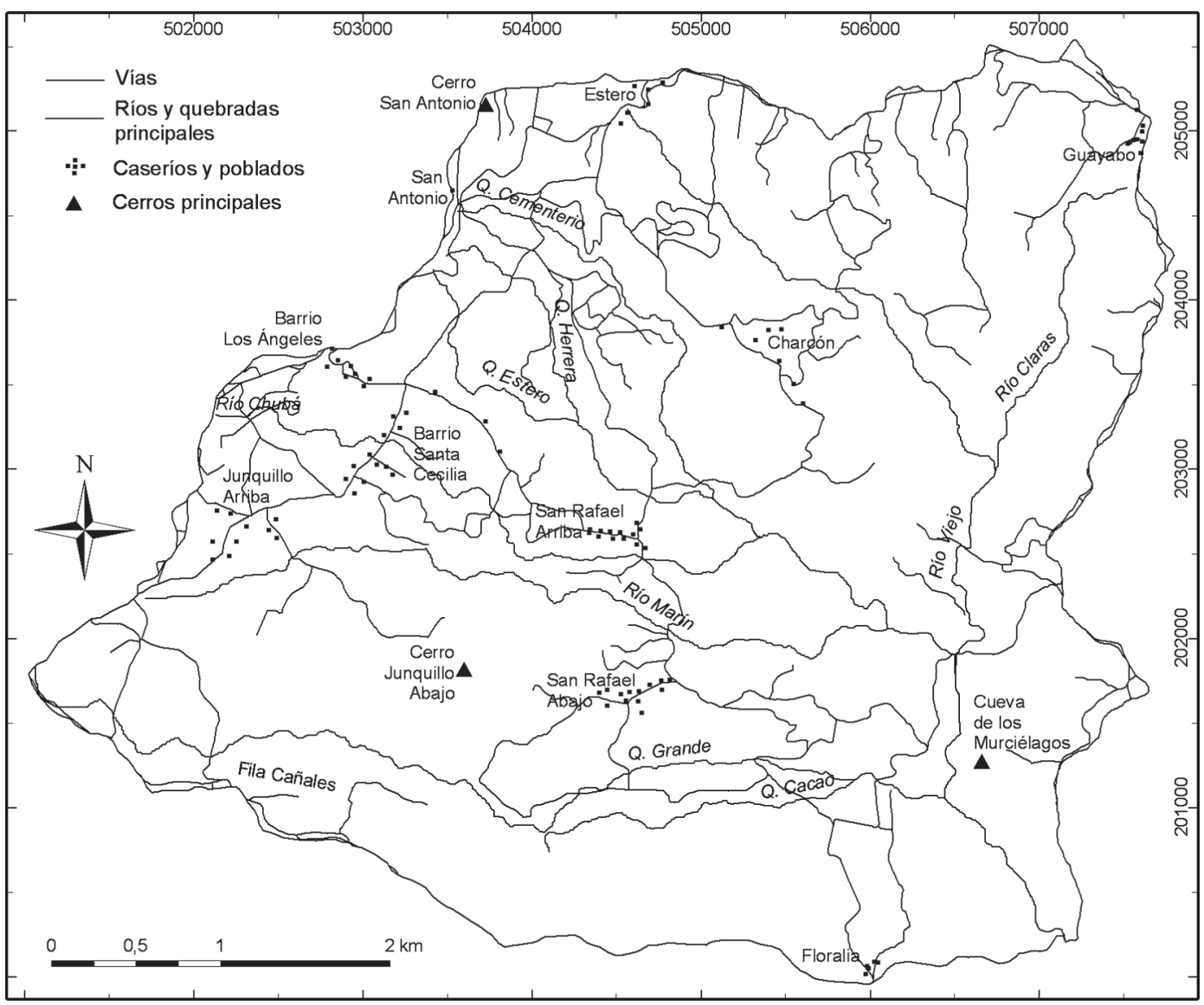

Fig. 2: Principales poblados y rasgos geográficos de la cuenca del río Viejo.

citarse: (1) la edad del deslizamiento, (2) la calidad y escala de las fotografías aéreas y los mapas base, (3) la complejidad morfológica y geológica del área de estudio, (4) los tipos y cambios de uso de la tierra, y (5) el grado de experiencia del geomorfólogo involucrado.

Para este estudio, la escala y la complejidad morfológica del área fueron los principales factores que dificultaron la delimitación de algunas coronas de deslizamientos identificados, así como de algunos depósitos de deslizamiento, limitando de esta forma la precisión del mapa de inventario.

La escala se definió a partir de la menor escala de las fotografías aéreas utilizadas en la región (1:40 000) y a la escala de la cartografía básica (1:25 000), porque a pesar de haberse hecho un trabajo de campo detallado y de contar con fotografías aéreas con mayor escala, desafortunadamente éstas no cubrían toda el área de estudio. Es por esto que, de acuerdo con la clasificación presentada por Malamud (2004), nuestro inventario es considerado como de mediana escala, por encontrarse en un rango de 1:25000 a 1:200 000 .

Por otro lado, la complejidad morfológica en el área es causada por la intensidad de los procesos de remoción en masa, que en su mayoría son generados por temporales, huracanes, la acción antrópica y la actividad sísmica, sobre litologías susceptibles a la erosión intensa. Al respecto, es importante mencionar que gran parte de los deslizamientos presentes en el área, fueron generados algunos de ellos y otros reactivados a partir de los enjambres sísmicos que la afectaron entre mayo 


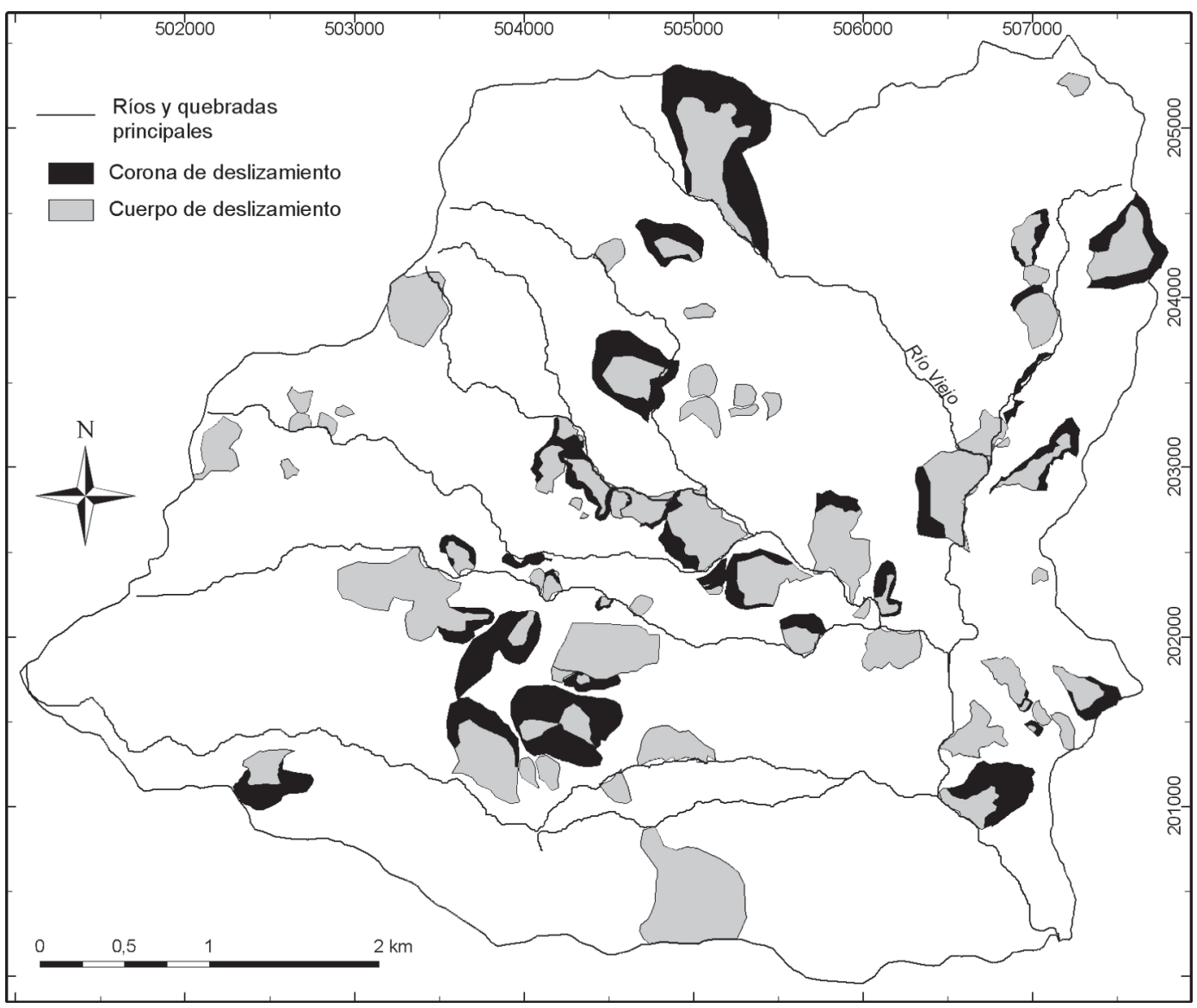

Fig. 3: Mapa inventario de deslizamientos.

y diciembre de 1990, y posiblemente controlados por algunos sistemas de alineamientos, identificados como las expresiones topográficas de las fuentes sísmicas responsables de dichos enjambres (Montero et al., 1991).

\section{GEOMORFOLOGÍA}

El mapa geomorfológico permite representar la dinámica y la complejidad morfológica de la cuenca, ya que a diferencia del mapa de inventario, en el que se marcan los deslizamientos como unidades independientes, en éste se presentan algunos de los deslizamientos dentro de áreas marcadas como afectadas por movimientos en masa. $\mathrm{Al}$ presentar los deslizamientos como procesos, se convierte en una mejor aproximación al mapa de susceptibilidad a deslizamientos que el mapa de inventario.

Para conocer la geomorfología del área, se llevaron a cabo observaciones de campo y se interpretaron las fotografías aéreas de los proyectos TERRA 1998 y CARTA 2005.

Las unidades geomorfológicas se clasificaron según su morfogénesis, de acuerdo con la propuesta de Van Zuidam (1986). En caso de que fuera necesario, estas unidades se subdividieron en rasgos morfológicos específicos. Así, el área fue dividida en nueve unidades principales, en las que predominan las formas de origen denudacional (códigos D1 a D7), que abarcan aproximadamente el 99\% del área de la cuenca. El 1\% restante corresponde con formas de origen estructural 
(códigos S1 y S2). Cada una de estas unidades se presenta en la figura 4 y se describen a continuación; las localidades y ríos mencionados en las descripciones, pueden observarse en la figura 2 .

\section{Formas de denudación}

\section{D1. Escarpes de denudación}

Corresponden con aproximadamente el 4\% del área total y están ubicados en diferentes sectores de la cuenca. Cuentan con una disección de baja a moderada, y en la mayor parte de esta unidad, las pendientes superan los $50^{\circ}$ de inclinación.
El escarpe más grande que conforma esta unidad se ubica en el sector oeste de la cuenca, inclinado hacia al este. Está ubicado al este de Santiago y tiene una altura promedio de $200 \mathrm{~m}$ y un largo de $2 \mathrm{~km}$. La pendiente promedio es de $24^{\circ}$, teniendo en cuenta que en algunos puntos alcanza pendientes de $62^{\circ}$ y que en el cálculo del promedio se incluyeron los depósitos coluviales. Abarca aproximadamente desde el sector donde nace el río Chubá, siguiendo por el límite occidental de la cuenca, hasta donde inicia el poblado conocido como San Antonio. Posee un sistema de drenaje paralelo, y su densidad varía de muy baja a moderada, con valores que oscilan entre 6,3 km/ $\mathrm{km}^{2}$ y 9,5 km/km² (Fig. 5 y Fig. 6). En 1990,

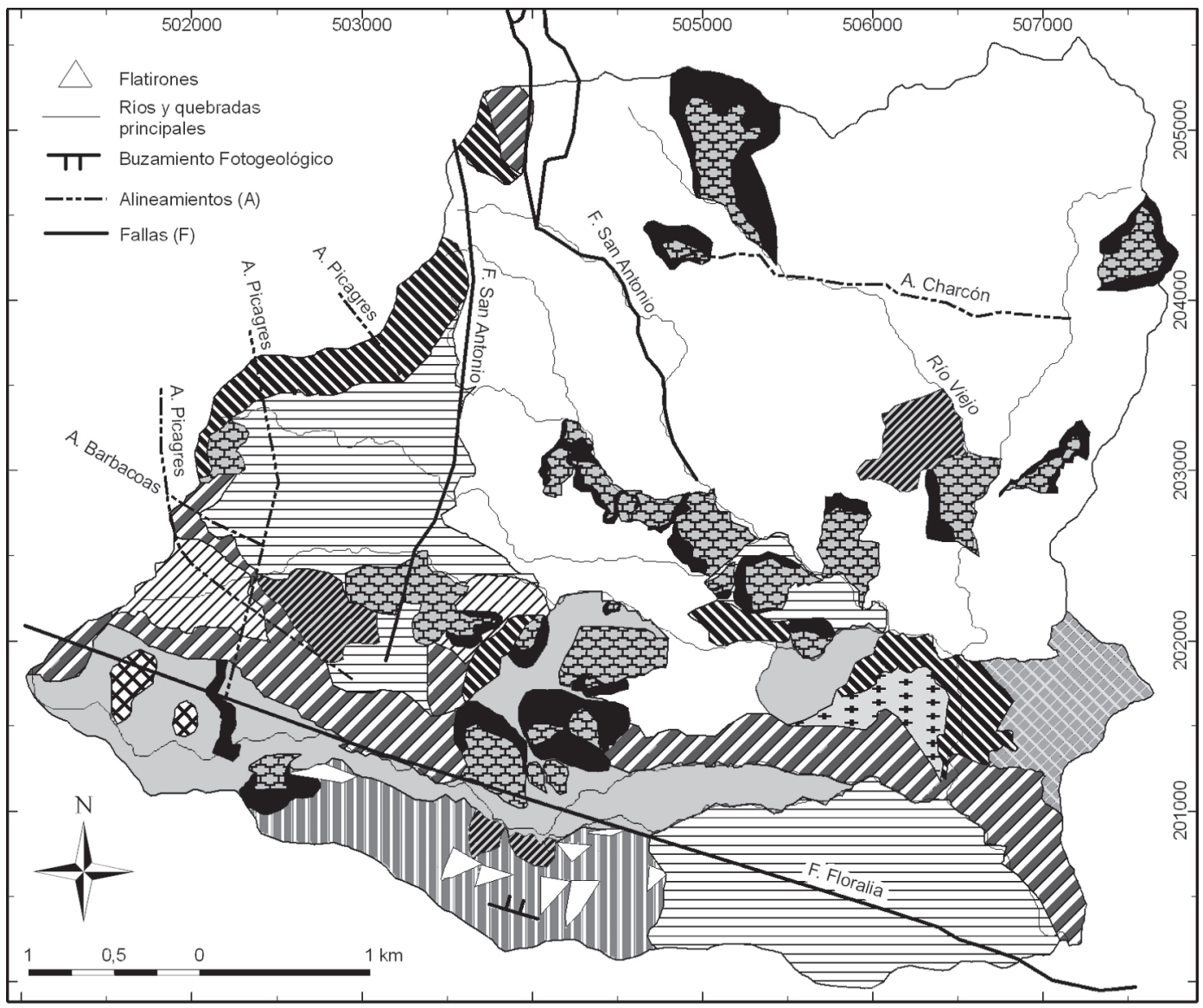

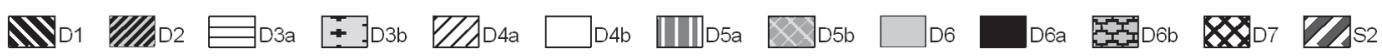

Fig. 4. Mapa geomorfológico del área de estudio. 


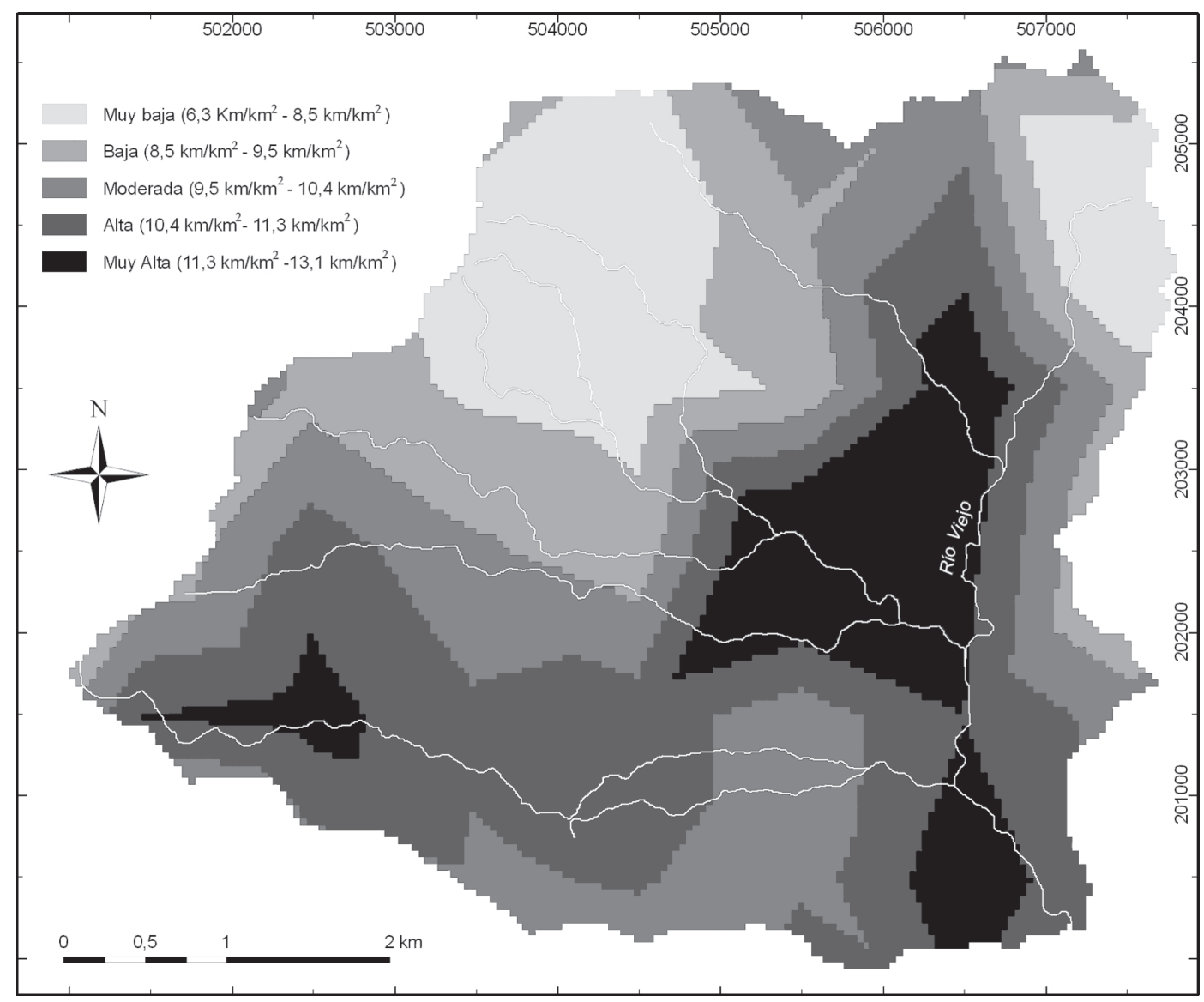

Fig. 5: Mapa de densidad de drenaje.

algunos sectores de dicho escarpe se reactivaron; uno de estos fue el sector denominado por Peraldo (1996) como "Deslizamiento Los Ángeles".

\section{D2. Laderas de piedemonte}

Abarcan aproximadamente un $2 \%$ de la cuenca, y se caracterizan por su relieve irregular con abultamientos. Son laderas con pendientes menores a los $15^{\circ}$ y con una disección escasa., formadas por acumulaciones de derrubios de las colinas denudacionales ubicadas a su alrededor, que están sometidas a intensos procesos de erosión, producto de pendientes altas, de una litología meteorizada, de períodos de precipitación intensa, y de la actividad sísmica.
Se localizan específicamente en tres puntos: al oeste del cerro Junquillo Abajo, al noreste de la cuenca, entre el río Viejo y las colinas ubicadas en el sector conocido como Charcón (Fig. 2), así como al sur de la quebrada Grande, en los alrededores de la coordenada 504000 E y $201000 \mathrm{~N}$.

\section{D3. Laderas de denudación de pendiente baja}

Corresponden aproximadamente al 16,6\% del área de la cuenca. Se caracterizan por tener laderas cortas y una topografía con un relieve moderadamente ondulado, donde predominan las colinas de baja altura con planicies de poca extensión. Pueden ser clasificadas en las siguientes sub-unidades: 


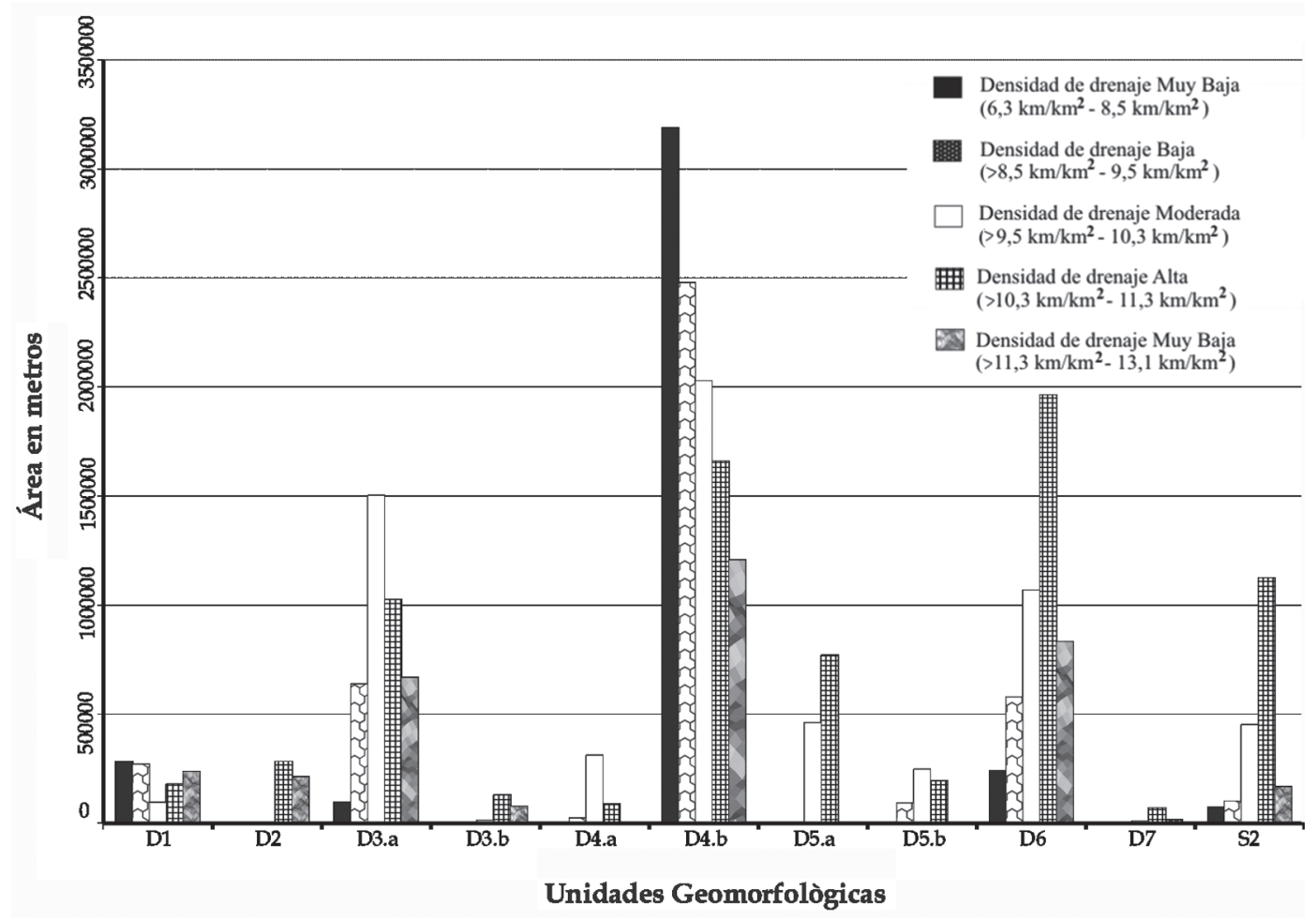

Fig. 6: Áreas de densidad de drenaje por unidad geomorfológica.

D3a. Áreas afectadas por reptación severa

Corresponden aproximadamente con el 15,6\% del área de la cuenca. El drenaje es paralelo y su densidad varía de baja y moderada, con valores que oscilan entre $6,3 \mathrm{~km} / \mathrm{km}^{2}$ y $10,3 \mathrm{~km} / \mathrm{km}^{2}$ (Fig. 5 y Fig. 6). Se ubica al sureste de la cuenca, en los alrededores del caserío de Floralia y también en el sector central-oeste, abarcando el barrio Los Ángeles y el barrio Santa Cecilia (Fig. 2).

Son áreas que, a pesar de contar con pendientes entre $\operatorname{los} 0^{\circ}$ y los $15^{\circ}$, presentan procesos de erosión intensa, caracterizadas por reptación severa y deforestación. Estos procesos pueden estar asociados a las características litológicas de la formación Grifo Alto, y a cambios en el uso de la tierra, ya que corresponden a áreas anteriormente utilizadas para cultivos de tabaco y arroz, y que actualmente están dedicadas a la ganadería.
D3b.Áreas afectadas por reptación leve

Esta unidad se encuentra al noroeste de la confluencia de la Quebrada Grande con el río Viejo, cubriendo únicamente un 1\% del área de la cuenca. Se distingue de la morfología de sus alrededores por las pendientes bajas, desde los $2^{\circ}$ hasta los $16^{\circ}$ aproximadamente, y cerca del $94 \%$ del área de esta sub-unidad cuenta con una densidad de drenaje clasificada entre alta y muy alta, que va desde $10,3 \mathrm{~km} / \mathrm{km}^{2}$ a $13,1 \mathrm{~km} / \mathrm{km}^{2}$ (Fig. 5 y Fig. 6)

\section{D4. Laderas de denudación de pendiente fuerte}

Abarcan aproximadamente el $44 \%$ del área de la cuenca. Se caracterizan por sus pendientes mayores a los $30^{\circ}$, laderas cortas, un sistema de 
drenaje dendrítico bien definido, y una densidad de drenaje que se clasifica principalmente de muy baja a moderada $\left(6,3 \mathrm{~km} / \mathrm{km}^{2}\right.$ a $\left.10,3 \mathrm{~km} / \mathrm{km}^{2}\right)($ Fig. 5 y Fig. 6). Pueden ser divididas en las siguientes sub-unidades:

D4a. Sub-unidad de divisorias angostas

Corresponde aproximadamente al 2\% del área de la cuenca. La característica principal de esta unidad son sus divisorias angostas, que responden a su fuerte pendiente y valles profundos. Se ubica en el sector conocido como Junquillo Arriba.

D4b. Sub-unidad de divisorias redondeadas

Esta sub-unidad abarca el $42 \%$ de la cuenca y se ubica al norte. Su característica más notable son sus valles en forma de $\mathrm{U}$ y sus divisorias redondeadas, que reflejan un relieve maduro. Posee pendientes que varían de $16^{\circ}$ a $55^{\circ}$ y un drenaje dendrítico. En ésta se encuentra la mayor parte de algunos de los principales caseríos, como el de San Rafael Abajo, Bellavista (ubicado en San Rafael Abajo), San Antonio, Charcón, El Estero, Guayabo (Fig 2).

\section{D5. Montañas y colinas de denudación con sistema de drenaje paralelo}

Corresponde al 7\% del área de la cuenca, y como su nombre lo indica, poseen un sistema de drenaje paralelo bien definido . Dentro de esta unidad se distinguen dos sub-unidades:

D5a. Intensamente disectadas

Localizadas en la zona central-sur de la cuenca, abarcan aproximadamente al 5\% del área total. Se caracterizan por tener las pendientes más altas de la cuenca, clasificadas principalmente entre $16^{\circ}$ y $55^{\circ}$. Representadas por una loma alargada en dirección NW-SE, cuya divisoria marca el borde de la cuenca. $\mathrm{Su}$ sistema de drenaje está formado por quebradas que fluyen en dirección NNE, que desembocan en el río Cacao, y aproximadamente el $64 \%$ de esta unidad cuenta con una densidad de drenaje clasificada como alta a muy alta (entre $10,3 \mathrm{~km} / \mathrm{km}^{2}$ y $13,1 \mathrm{~km} / \mathrm{km}^{2}$ ). La principal característica de esta unidad es la presencia de flatirones (Fig. 4), los cuales indican estratos de roca sedimentarias buzantes hacia el NNE.

\section{D5b. Moderadamente disectadas}

Abarcan aproximadamente el 2\% del área de la cuenca, y se caracterizan por la presencia de cárcavas grandes. Se ubican al sureste del río Viejo, aproximadamente en las coordenadas $507000 \mathrm{E}$ y 201500 N, en el sector conocido como "La Cueva de los Murciélagos" (Fig. 2). Sus pendientes varían principalmente entre $8^{\circ}$ y $16^{\circ}$. Esta sub-unidad cuenta con valles profundos, y presenta una densidad de drenaje que se clasifica principalmente en moderada a alta (de $9,5 \mathrm{~km} / \mathrm{km}^{2}$ a 11,3 km/ $\left.\mathrm{km}^{2}\right)($ Fig. 5 y Fig. 6), generando así un relieve moderadamente disectado. De igual forma, debido al fuerte proceso de erosión ocasionado por los cambios de uso de la tierra, actualmente esta área está cubierta por tacotal.

\section{D6. Áreas severamente afectadas por movimientos en masa}

A pesar de que, en general, toda la cuenca está afectada por movimientos en masa, se han clasificado dentro de esta unidad las áreas más críticas, que corresponden al 18,5\% del área total. Se localizan principalmente en los siguientes puntos:

$\mathrm{Al}$ este del cerro Junquillo Abajo, en el área abarcada entre la Quebrada Grande y el río Marín, que corresponde aproximadamente a los alrededores del punto con coordenadas 503800 E y $201800 \mathrm{~N}$ (Fig. 2).

En el valle entre la Quebrada Grande y el río Cacao (Fig. 2), el cual según Peraldo (1996), podría corresponder con una zona de inestabilidad asociada a la falla Floralia (Fig. 4), la cual generó movimientos en masa en forma de flujos de detritos en dirección W-E. En fotos aéreas esta área se ve como un único flujo de aproximadamente 4,5 km de largo y 300 metros de ancho, que este mismo autor describe como "con forma de lengua". 
Al noreste del cementerio de San Rafael y al sur del río Marín, en los alrededores del punto con coordenadas 505500 E y 201800 N (Fig. 2).

Son áreas que se caracterizan por bloques de terreno basculados a causa de movimientos rotacionales, afectadas por reptación intensa, cárcavas profundas, flujos de detritos y deslizamientos que cortan otros deslizamientos. Son comunes los deslizamientos rotacionales y de movimiento lento en las zonas de mayor pendiente, aunque también se presentan deslizamientos superficiales. El movimiento en esta zona no está directamente relacionado a las pendientes, ya que éstas varían desde $0^{\circ}$ hasta $35^{\circ}$; sin embargo, sí podría estar relacionado con los cambios de uso de la tierra, y con las características litológicas de la Formación Grifo Alto presente en esta área, ya que de acuerdo con Peraldo (1996), la meteorización y la alteración hidrotermal de ésta Formación produce suelos residuales de mucho espesor, con contenidos arcillosos importantes que llegan a ser inestables en presencia del agua.

Una característica que resalta en esta unidad es que su densidad de drenaje se clasifica principalmente entre moderada y muy alta (de $9,5 \mathrm{~km} / \mathrm{km}^{2}$ a 13,1 km/km²) (Fig. 5 y Fig. 6).

Dentro de esta unidad se distinguen dos subunidades que se describen de forma independiente:

D6a. Sub-unidad de escarpes de deslizamientos

Esta sub-unidad abarca el 4\% del área de la cuenca, aproximadamente. Está conformada por los escarpes de las coronas principales o laterales de los deslizamientos principales del área, ya sean inactivos o activos. En general, posee pendientes entre $35^{\circ}$ y $55^{\circ}$; aunque hay algunos escarpes con pendientes entre los $8^{\circ}$ y los $16^{\circ}$.

Son áreas muy inestables con respecto a otras unidades, por lo que es común identificar rasgos fotogeológicos formados por retrocesos de escarpes y la ampliación del área afectada por la reptación. Sin embargo, de acuerdo con comprobaciones hechas en campo, también se encontraron algunos escarpes que pertenecen a deslizamientos antiguos que actualmente se encuentran inactivos. Un ejemplo de estos escarpes es el ubicado en el sector del Estero (Fig. 2), que es el más grande de la cuenca, con cerca de $150 \mathrm{~m}$ de altura y un área aproximada de 29 ha.

Cabe destacar el escarpe ubicado al sur del sector conocido como Junquillo Arriba (Fig. 2), que coincide con uno de los alineamientos de la falla Picagres (Fig. 4), y que en el campo puede verse como el inicio de los flujos de detritos que se mencionó ocurrieron en el valle de la Quebrada Grande y el río Cacao.

D6b. Sub-unidad de depósitos de deslizamiento

En esta sub-unidad se agruparon los principales depósitos de algunos deslizamientos identificables en las fotografías aéreas. Corresponden al 6,5\% del área de la cuenca. No están relacionados con un tipo de pendiente específico, ya que presentan pendientes tanto bajas como elevadas.

La mayor parte de esta unidad corresponde al deslizamiento de San Rafael Arriba, ubicado al norte del caserío del mismo nombre (Fig. 2). Este deslizamiento es generado principalmente por el cambio de uso de la tierra y por el socavamiento intenso que producen al pie del área inestable los ríos Chubá y Marín (Fig. 2).

Otro depósito de gran volumen, es el que corresponde al del deslizamiento ocurrido en el flanco oeste del cerro Junquillo Abajo (Fig. 2).

\section{D7. Cerros Relictos}

Localizados al suroeste de la cuenca, entre la quebrada Grande y el río Cacao, abarcan aproximadamente el $0,4 \%$ de la cuenca. Son dos cerros que sobresalen por su altura; uno de estos tiene forma redondeada con un diámetro aproximado de 180 $\mathrm{m}$, y el otro tiene forma ovoidal, cuyos ejes miden aproximadamente 225 y $450 \mathrm{~m}$.

En general, las pendientes dentro de esta unidad son mayores a los $16^{\circ}$. La ubicación de estos cerros coincide con lo que se interpreta como la zona de arranque de los flujos de detritos mencionados dentro de las áreas severamente afectadas por movimientos en masa. 
De acuerdo con sus características y su ubicación, se podrían plantear dos hipótesis sobre su origen: la primera es que se trata de megafragmentos que fueron arrastrados por los flujos, y la segunda, que son cerros testigos, es decir, cerros formados por rocas poco erosionables que quedan precisamente como testigo de una capa que ha desaparecido por erosión de los alrededores.

\section{Formas de origen estructural}

\section{S1. Alineamientos}

Existen varios alineamientos en el área (Fig.4), tales como el sistema de fallas San Antonio, el sistema de fallas Picagres, al alineamiento Barbacoas, y la falla Floralia, los cuales ya habían sido descritos por Montero et al. (1991), y que se encuentran asociados a algunos de los escarpes presentes en la cuenca, que se describirán más adelante.

Dentro del área abarcada por la cuenca, el alineamiento correspondiente a la falla San Antonio alcanza una longitud aproximada de $3 \mathrm{~km}$, llegando hasta el oeste de San Rafael Abajo. De acuerdo con Montero et al. (1991), esta falla se hace evidente en ambos flancos del cerro San Antonio (Fig. 2) del siguiente modo:al oeste del cerro, la falla cambia su rumbo de SE a SW, lo que permite inferir que el medio está sometido a un par de fuerzas simples dextrales; y al este del cerro, se identificaron dos ramales que forman una cuenca de tracción producto del sistema dextral en la zona. El alineamiento del flanco este sigue haciéndose evidente con un rumbo $\mathrm{SE}$, ya que prácticamente el cauce de la quebrada Cementerio (Fig. 2) se ha adaptado a éste.

De igual forma, se encontró un nuevo alineamiento denominado Charcón, el cual fue trazado de acuerdo con la interpretación de las fotos aéreas, en las que se puede notar un alineamiento sistemático de las quebradas de la zona, y un cambio de SE a E en el rumbo del río Viejo.

El alineamiento Charcón tiene una dirección oeste-este y va aproximadamente desde las coordenadas $504800 \mathrm{E} / 204200 \mathrm{~N}$ hasta 507100 E / $203900 \mathrm{~N}$, lo cual corresponde con el sector conocido como Charcón (de ahí su nombre).

\section{S2. Escarpes estructurales}

Esta unidad se diferenció principalmente por la inclinación de las pendientes, que oscilan entre los $35^{\circ}$ y los $85^{\circ}$, por un patrón de drenaje subparalelo , y por la escasa vegetación. Abarcan aproximadamente el 7,5\% del área de la cuenca, y se localizan en los sectores suroeste, norte y sureste (Fig. 4).

En el sector suroeste, el escarpe se inicia al sur del cerro Junquillo Abajo, y sigue con un rumbo NW por cerca de $1,5 \mathrm{~km}$, hasta bifurcarse hacia el NW y el W. La primera ramificación presenta una inclinación hacia el sur, mantiene su rumbo NW por $1 \mathrm{~km}$, para luego cambiar a NE, rodeando el poblado de Junquillo Arriba. Tiene una altura promedio de $100 \mathrm{~m}$ y una longitud de $1300 \mathrm{~m}$. El escarpe correspondiente al segundo ramal, está inclinado hacia el noreste y mantiene su rumbo E-W por espacio de $1 \mathrm{~km}$, para luego cambiar hacia el NE-SW, siguiendo el borde de la cuenca. Posee una altura promedio de $150 \mathrm{~m}$ y una longitud de $1,5 \mathrm{~km}$. El extremo W de este escarpe colinda con el extremo sur de otro pequeño, el cual corresponde con el flanco suroeste del cerro Junquillo, con una longitud aproximada de $370 \mathrm{~m}$, una altura de $200 \mathrm{~m}$ y un rumbo N-S.

De acuerdo con Montero et al. (1991), estos escarpes que han estado relacionados con la formación de los flujos de detritos y con las fallas mencionadas, podrían tener un origen tectónico, de acuerdo con los rumbos de los alineamientos de Barbacoas, Floralia y Picagres (Fig. 4).

En el sector norte se identifica un escarpe en la ladera este del cerro San Antonio. Tiene un ancho aproximado de $500 \mathrm{~m}$, una altura aproximada de $220 \mathrm{~m}$ y una inclinación hacia el este. Coincide con el rumbo del alineamiento principal del sistema de fallas San Antonio y se caracteriza por tener una densidad de drenaje muy baja, de 6,3 $\mathrm{km} / \mathrm{km}^{2}$ a $8,5 \mathrm{~km} / \mathrm{km}^{2}$ (Fig. 5 y Fig.6). 
Finalmente, en el sector sureste, se localiza un escarpe que se inicia aproximadamente en las coordenadas $504400 \mathrm{E} / 201300 \mathrm{~N}$ y que se extiende por $3 \mathrm{~km}$ a lo largo de la quebrada Grande y el río Viejo, hasta alcanzar la desembocadura de este último. Este escarpe, que está asociado al contrabuzamiento de las laderas adyacentes, tiene una altura promedio de 200 metros y está inclinado hacia el sur.

\section{DISCUSIÓN Y CONCLUSIONES}

El mapa geomorfológico permite tener un conocimiento aproximado de las condiciones específicas bajo las cuales ocurren diferentes tipos de deslizamientos en el área, además que ayuda a entender su morfodinámica. Para el caso de la cuenca del río Viejo, el 99\% del área total, ha sido clasificada dentro de unidades geomorfológicas de origen denudacional, lo que evidencia su dinámica activa, que corresponde a lo que Mora et al (1990) describe como la fase juvenil en la que se encuentra dentro del ciclo geomorfológico de erosión.

Las unidades geomorfológicas clasificadas como "severamente afectadas por movimientos en masa", corresponden al 18,6\% del área total de la cuenca, y son áreas críticas con diferentes procesos y formas de movimientos en masa. Una de las características de esta unidad es que aproximadamente el $70 \%$ de su área cuenta con pendientes bajas (entre $0^{\circ}$ y $16^{\circ}$ ), refutando la hipótesis de que la pendiente es un factor condicionante para la ocurrencia de deslizamientos.

Dichas áreas clasificadas como severamente afectadas por movimientos en masa y que tienen pendientes bajas, corresponden a áreas de depósito de material generado por antiguos procesos de remoción en masa en laderas adyacentes, como es el caso del área que corresponde al valle comprendido entre el río Cacao y la quebrada Grande, que de acuerdo con Peraldo (1996) se vio afectada por movimientos en masa que formaron flujos y que es bien identificable en fotointerpretación. El descubrimiento de este tipo de procesos ocurridos en el área, evidenció la importancia de la inclusión de la geomorfología como variable a tener en cuenta dentro del análisis de la susceptibilidad, y de otros factores condicionantes para la ocurrencia de deslizamientos como la litología y las características hidrogeológicas.

Por otro lado, si se compara el mapa geomorfológico con el mapa de inventario de deslizamientos, se hace evidente que el primero resultaría más completo para un análisis de la susceptibilidad a deslizamientos. Esto se debe a que el mapa geomorfológico incorpora todas las formas de remoción en masa presentes, incluso los detalles -si estos pueden fotointerpretarse o verse fácilmente en campo-; mientras que en el segundo solo se incluyen los deslizamientos, y solo aquellos cuya delimitación fue posible, ya que por tratarse de un área tan activa muchas coronas de deslizamiento no fueron fáciles de identificar, lo que se convirtió en una limitante.

Sin embargo, es claro que cada uno de estos mapas tendrá diferente participación en la zonificación de la susceptibilidad: el mapa geomorfológico a partir de la ponderación de sus unidades se tomaría en consideración en metodologías heurísticas; mientras que, el mapa de inventario permitiría confirmar los resultados arrojados con la aplicación de metodologías heurísticas, y también se tomaría en consideración en la aplicación de métodos estadísticos para el cálculo de la susceptibilidad. No obstante, el uso del mapa de inventario debe tener en cuenta la limitante mencionada.

Respecto a la litología del área, se cuenta únicamente con el mapa realizado a nivel regional por Denyer \& Arias (1990), cuya escala presentaba una litología muy homogénea -casi el 85\% del área pertenece a la formación Grifo Alto- y con comportamiento similar en el área de estudio. Es por esto, que para la zonificación de la susceptibilidad por deslizamiento se debe sustituir este factor por la geomorfología, el cual ya ha sido utilizado en otros trabajos como el de Camacho et al. (2004) y Bonilla (2007) al considerar que la litología no es un elemento que contribuye en el desarrollo de una zonificación.

Por otro lado, al relacionar las tres unidades litológicas presentes en la cuenca con las diferentes clases de densidad de drenaje, se encontró que 
la distribución entre las densidades de drenaje y las unidades geológicas es uniforme, y que no existe una relación directa entre unidades geológicas y densidades de drenaje específicas.

Del mismo modo, la comparación del mapa de densidad drenajes con el mapa geomorfológico, permitió concluir lo siguiente:

1. Respecto a unidades cuyas áreas se clasifican principalmente con densidades de drenaje clasificadas entre altas y muy altas $\left(10,3 \mathrm{~km} / \mathrm{km}^{2}\right.$ a $13,1 \mathrm{~km} / \mathrm{km}^{2}$ ), sobresalen las laderas de piedemonte, con el $99,9 \%$ de su área, la sub-unidad de laderas de denudación de pendiente baja levemente afectadas por reptación, con el $94 \%$ de su área, y los cerros relictos con un $89 \%$ de su área.

2. El $83 \%$ del área definida como severamente afectada por movimientos en masa cuenta con una densidad de drenaje entre Moderada y Muy Alta $\left(9,5 \mathrm{~km} / \mathrm{km}^{2}\right.$ a $\left.13,1 \mathrm{~km} / \mathrm{km}^{2}\right)$.

3. La unidad de laderas de denudación de pendiente fuerte sobresale con un $73 \%$ de su área clasificada con densidad de drenaje de muy baja a moderada $\left(6,3 \mathrm{~km} / \mathrm{km}^{2}\right.$ a $\left.10,3 \mathrm{~km} / \mathrm{km}^{2}\right)$.

Las anteriores conclusiones permiten plantear como hipótesis que los valores altos de densidad de drenaje condicionan la ocurrencia de deslizamientos. Sin embargo, es necesario resaltar que esta hipótesis solo puede ser confirmada a partir de estudios geotécnicos e hidrogeológicos.

Por otro lado, se encontró que la reptación es una de las formas de movimiento en masa característica en la cuenca, y de acuerdo con Mora (1985), evidencia una variación importante de cantidad de agua generada por las temporadas de lluvias, que produce expansiones y retracciones de la capa superficial de suelo, originando los esfuerzos, primero perpendiculares y luego paralelos a la superficie que son evidentes en campo.

Finalmente, se concluye que este estudio permitió reconocer la importancia de la caracterización geomorfológica como procedimiento clave para entender la dinámica histórica de las formas en el área de estudio y de los eventos de deslizamiento que se han presentado, con el fin de validar variables y metodologías a tener en cuenta en la zonificación de la susceptibilidad por deslizamiento.

\section{RECOMENDACIONES}

Teniendo en cuenta que una de las grandes limitaciones que generalmente se presentan al hacer un estudio de susceptibilidad por deslizamientos es la falta de una buena base de datos de la ocurrencia histórica de deslizamientos, se recomienda para áreas relativamente pequeñas, como esta cuenca, mantener actualizada el mapa de inventario de deslizamientos a partir de un trabajo de campo integral que incluya la información suministrada por la comunidad.

Una de las dificultades relacionadas con la actualización del mapa de inventario de deslizamientos es la falta de delegación de esta responsabilidad a una entidad específica; esto a pesar de su importancia en las labores de prevención, y de la legislación actual costarricense, según la cual, los gobiernos locales deben trabajar en los temas de prevención. En el caso de Costa Rica, generalmente los inventarios de deslizamiento se elaboran dentro de proyectos de investigación desarrollados por la escuela de Geología, o de Ingeniería Civil de la Universidad de Costa Rica, que debido a su naturaleza cuentan con una duración limitada, después de la cual la base de datos de deslizamiento no se actualiza.

Por lo anterior, también se recomienda plantear un proyecto de organización comunitaria enfocado en la búsqueda de empoderamiento de la comunidad y las instancias gubernativas y privadas cuya actividad se afecta con la ocurrencia de deslizamientos en el área, para establecer redes de monitoreo que permitan la actualización de las bases de datos de deslizamiento en la cuenca, rescatando la memoria histórica comunal e individual.

Teniendo en cuenta las limitaciones de información espacial histórica y del detalle que requería este trabajo, y de acuerdo con lo planteado por Ibsen \& Brunsden (1996), se concluye que es muy difícil obtener un mapa de inventario de deslizamiento que esté completo con respecto tanto al área cubierta como al período de tiempo investigado, por lo que se recomienda garantizar la información espacial (como fotos aéreas o imágenes de satélite) actualizadas con mayor 
frecuencia, para obtener por lo menos inventarios de deslizamientos para periodos fijos, relacionados a las imágenes disponibles.

De igual forma, debido a que el área fue incorporada únicamente en un estudio geológico regional a escala 1:50000 realizado por Denyer y Arias (1990), se recomienda hacer un estudio detallado de la litología de los cerros relictos, ya que en el mapa actual únicamente se puede ver que pertenece a la información Grifo Alto, pero no hay información que permita determinar cuál de las hipótesis planteadas sobre su origen en este artículo es la verdadera.

\section{AGRADECIMIENTOS}

La presente investigación forma parte del proyecto de tesis de Ángela Marcela Suárez Castiblanco, para optar por la Maestría académica en Gestión del Riesgo en Desastres y Atención de Emergencias del Posgrado Centroamericano en Geología de la Universidad de Costa Rica. Además, se desarrolló en el marco del Proyecto de Investigación $\mathrm{N}^{\circ} 113$ A6-076, titulado Aplicación de Sistemas de Información Geográfica y Percepción Remota a la zonificación de inestabilidad de laderas, área central del país, inscrito en la Vicerrectoría de Investigación.

Se agradece al Lic. Luis Guillermo Salazar Mondragón y al M.Sc. Rolando Mora Chinchilla, por sus sugerencias y aportes, y a la geóloga Diana Medina Sánchez, por su colaboración como asistente del proyecto.

De igual forma, se agradece a la comunidad de San Rafael por su amable disposición y valiosos aportes durante el trabajo de campo, en especial a doña Chola, a don Rodrigo Cerdas, a doña Aminta, a Silvia Masis, a don Elías Masis, a Gabriela Masis, a Álvaro, a don Antonio Fallas, a doña Stellia, a don Elpidio Bermúdez, a Melissa Bermúdez, a doña María Eugenia Otárola, a Violeta Fallas, a don Froylan Mora, a doña Maria Luisa, y al futuro geólogo Willy Salazar.

\section{REFERENCIAS}

BONILLA, E., 2006: Estudio diagnóstico de amenazas asociadas a geodinámica externa en la parte media de la cuenca del río Limón, cantón de Buenos Aires, Puntarenas.-170 págs. Univ. de Costa Rica, San José [Tesis Lic.].

CAMACHO, D., CHAVES, J., MURILLO, D., 2004: Diagnóstico de la susceptibilidad al deslizamiento e inundación, análisis del contexto sísmico y reconocimiento de los peligros volcánicos para el cantón de Montes de Oca: Una contribución al plan regulador del municipio.- 105 págs. Univ. de Costa Rica, San José [Tesis de Lic.].

CARRARA, A., CARDINALLI, M., GUZZETTI, F. \& REICHENBACH, P., 1995: GIS technology in mapping landslide hazard.- En: CARRARA, A. \& GUZZETTI, F. (eds.): Geographical Information Systems in assessing natural hazards.- Kluwer Academic Publishers, Holanda.

DENYER, P \& ARIAS, O., 1991: Estratigrafía de la región central de Costa Rica.- Rev. Geol. Amér. Central, 12: 1-59.

IBSEN, M. \& BRUNSDEN, D., 1996: The nature, use and problems of historical archives for temporal ocurrente of landslides, with specific referente to the south coast of Britain, Ventnor, Isle of Wight.- Geomorphology, 15(3-4): 241-258.

INEC, 2000: Censos de población y vivienda.http://www.inec.go.cr/ [Consulta: 15 de setiembre de 2008].

MALAMUD, B., TURCOTTE, D., GUZZETTI, F. \& REINCHENBACH, P., 2004: Landslide inventories and their statistical properties.- Earth Surface Processes and Landforms, 29(6): 687-711. 
MONTERO, W., ROJAS, W., BOSCHINI, I., BARQUERO, R., FLORES, H., 1991: Neotectónica de la región de Puriscal, origen de la sismicidad de Mayo-Diciembre de 1990.- $5^{\text {to }}$ Seminario Nacional de Geotecnia, San José, Costa Rica: 4-38 a 4-51.

MORA, S., 1991: La dinámica del deslizamiento de Puriscal, Costa Rica.- $5^{\text {to }}$ Seminario Nacional de Geotecnia, San José, Costa Rica: 2-1 a 2-20.

MORA, S., 2007: The nature of disasters: Reflections on their causes and consequences in latin america and the caribbean (LAC).Conferencia Magistral, $1^{\text {st }}$ North American Landslide Conference, American Association of Environmental and Engineering Geologist; Vail Colorado, June 3-8.

MORA, R., MORA, S. \& VAHRSON, W., 1992: Macrozonificación de la amenaza de deslizamientos y resultados obtenidos en el área del valle central de Costa Rica.- Escala 1:286 000, CEPREDENAC, San José

PERALDO, G., 1996: Amenaza de deslizamientos, sector central del cantón de Puriscal y problemática de la Ciudad de Santiago.- 210 págs. Univ. de Costa Rica, San José [Tesis Lic.].

VAN WESTEN, C. \& SOETERS, R., 2000: Remote Sensing and Geographic Information Systems for Natural Disaster Management.- En: ROY, P.S., VAN WESTEN, C.J., JHA, V.K., LAKHERA, R.C. \& CHAMPATI RAY, P.K. (eds.): Natural Disasters and their Mitigation. A Remote Sensing and GIS Perspective.Indian Institute of Remote Sensing, National Remote Sensing Agency, 31-76.

VAN ZUIDAM, R.A., 1986: Aerial Photointerpretation in terrain analysis and geomorphologic mapping.- 442 págs. Ed. Smits Publishers, The Hague. 
